\title{
ACOUSTOELECTRICAL RELAXATION IN GaAs BY THE FORMATION AND THE RECOVERY OF METASTABLE EL2*
}

\author{
J. Ertel, H.G. Brion and P. HaAsen \\ Institut für Metallphysik, Universität Göttingen \\ Hospitalstr. 3-7, W-3400 Göttingen, Germany \\ Dedicated to Professor Dr. Julian Auleytner \\ on the occasion of his 70th birthday
}

(Received August 24, 1992)

\begin{abstract}
The transformations between the normal and metastable state of EL2 in $\mathrm{GaAs}$ are investigated. We apply the internal friction technique as a probe for very small conductivities and discuss the changes in conductivity by the formation and recovery of EL2*. At $40 \mathrm{~K}$ both shallow acceptors and EL2 are photoquenched into neutral metastable states. The acceptors are likely to be associated with EL2* forming an electrically inactive state. We identify two damping peaks at 60 and $95 \mathrm{~K}$ with the regeneration of the acceptors and EL2 respectively. The latter indicates a regeneration via the charged defect $(\mathrm{EL} 2)^{+}$.

PACS numbers: $62.40 .+\mathrm{i}, 77.40 .+\mathrm{i}, 72.80 . \mathrm{Ey}, 71.55 . \mathrm{Eq}$
\end{abstract}

\section{Introduction}

EL2 is an intrinsic midgap defect in GaAs. Due to its presence technologically important semi-insulating (SI) material can be achieved. If the concentration of shallow donors is smaller than that of shallow acceptors, this surplus is compensated by electrons from the deep donor EL2. As long as some neutral EL2 defects are present, the Fermi level is pinned near the middle of the gap by the remaining EL2 defects.

Various investigations have been performed to study the properties of EL2. The proposed structure models have led to controversial pictures concerning the configuration of the involved point defects (e.g., Refs. [1-5]). There is agreement with respect to the participation of an arsenic antisite $A s_{\mathrm{Ga}_{a}}$. Another important feature is the appearance of the metastable state named EL2* ${ }^{*}$ It is formed at low temperatures under illumination with light of a maximum quenching efficiency 
at an energy of $1.13 \mathrm{eV}$ [6]. The recovery back to the normal state occurs at temperatures above $120 \mathrm{~K}$ [6]. The quenched state is electrically inactive and not accessible by most experimental methods [7]. Thus, very little is known about this metastable state, and only the transitions between the two states have been investigated. Techniques like photoconductivity [8], infrared absorption [9], and photocapacitance [6] have been applied to study the transformation. In this paper we present another experimental method, based on the acoustoelectrical relaxation of conduction electrons screening the piezoelectrical field of harmonically strained GaAs crystals. The coupling of the electrons to an ultrasonic wave can be detected by an acoustic damping, i.e. internal friction (IF). The advantage of this method is its high sensitivity to very small conductivities and the high time resolution without the need to use any electrical contact. The capture and the release of electrons during the transformations between the normal and the metastable state of EL2 are monitored. For these investigations high resistivity samples are required.

Both theoretical and experimental investigations of the interaction between acoustic waves in piezoelectric semiconductors and conduction carriers have been performed by several authors ([10-12] and others). The experimental works present conductivity measurements in SI crystals by the IF method. Concerning the relaxation time there is some controversy between theory [10] and experimental data [12]. In Sec. 3.1 we first show results achieved at high temperature to clarify this problem. In Sec. 3.2 we describe the low temperature results. The damping peaks achieved by irradiation with a Nd doped yttrium aluminium garnet (Nd:YAG) laser and the heating curves after irradiation are analyzed in Sec. 4.

\section{Experimental procedures}

\subsection{Internal friction method}

Anelastic relaxation processes leading to an attenuation of a vibrating crystal can be detected as IF. Such a process is the screening of piezoelectric fields by conduction electrons. Depending on the efficiency of the acoustoelectric coupling, the loss of energy per cycle, $Q^{-1}$, is given by an equation derived by Zener [13]:

$$
Q^{-1}=\Delta \frac{\omega \tau}{1+(\omega \tau)^{2}}
$$

In this equation $\omega=2 \pi \nu$ is the circular frequency of the vibration, $\tau-$ the characteristic time for the screening of the electric field and $\Delta=e_{14}^{2} / \varepsilon c-$ the relaxation strength. $\varepsilon$ is the dielectric constant, $e_{14}$ and $c$ respectively the appropriate piezoelectric and elastic constants in a cubic crystallographic system. For a thermally activated process $\tau$ can be expressed by an Arrhenius equation

$$
\tau=\tau_{0} \exp (E / k T)
$$

where $E$ is the activation enthalpy, $k-$ Boltzmann's constant, and $T-$ the temperature. For a dielectric relaxation according to Maxwell $\tau$ is given by

$$
\tau=\tau_{\mathrm{M}}=\varepsilon / \sigma
$$

where $\sigma$ is the electrical conductivity. Both the mobility and the temperature dependence of the concentration of conduction electrons contributing to the conductivity can be obtained by Hall effect and conductivity measurements to verify a Maxwell relaxation time. We come to this point in Sec. 3.1. 
Similar to the changes of $Q^{-1}$ due to the screening of piezoelectric fields corresponding variations of the resonance frequency on account of a reverse piezoelectric effect can be observed. If the electric field is unscreened, the sample appears to be stiffer. For $\omega \tau \gg 1$ at low temperatures the relaxation time given by Eq. (2) is too large to allow an efficient screening of the electric field. At high temperatures $(\omega \tau \ll 1)$ the field is screened very quickly. In this case the resonance frequency $\nu$ decreases. An equation analogous to (1) for the relative change $\delta \nu / \nu$ is given by [14]:

$$
\frac{\delta \nu}{\nu}=\frac{\Delta}{2} \frac{(\omega \tau)^{2}}{1+(\omega \tau)^{2}}
$$

The experimental data were obtained using the composite oscillator technique as developed by Schwarz [15]. The sample is glued to a piezoelectric quartz transducer as shown in Fig. 1 and stimulated to vibrate in a pure longitudinal vibration mode at its mechanical fundamental frequency of $106 \pm 2 \mathrm{kHz}$. The strain amplitude is
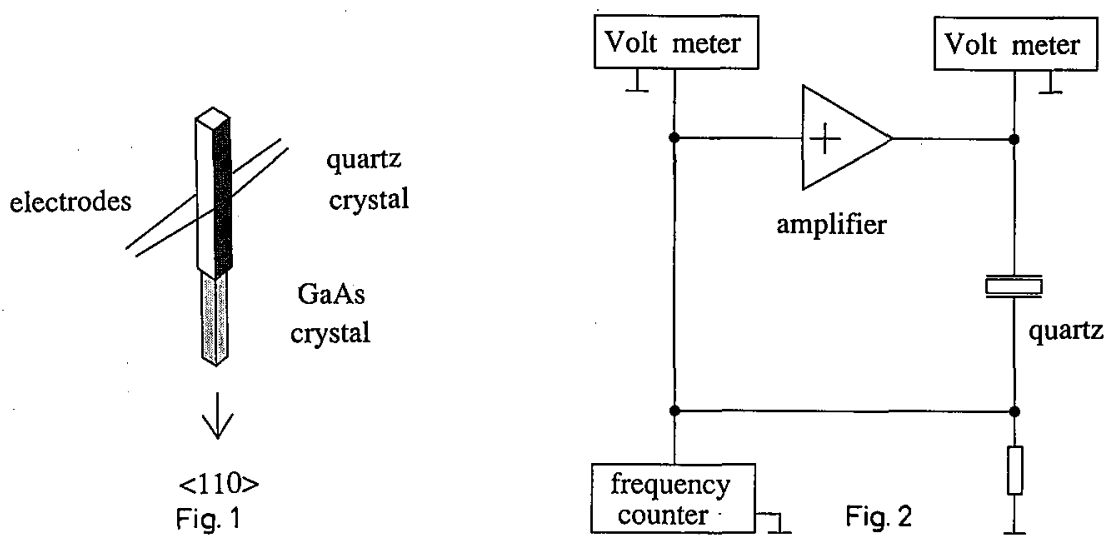

Fig. 1. Composite oscillator: the quartz crystal is fixed and contacted by two tinned copper wires.

Fig. 2. Schematic diagram of the feedback circuit.

kept constant at $1.0 \times 10^{-6}$ in a feedback circuit (Fig. 2). The IF was determined from the gain of the amplifier, which is a measure for the dissipated energy in the crystal. The investigated temperature range was between 40 and $400 \mathrm{~K}$.

Nd:YAG laser $(1.17 \mathrm{eV})$ was used to study the influence of light on the relaxation time. These experiments are necessary to understand the results of quenching. A power of a few $\mathrm{mW}$ was applied to the sample via a light pipe. Quenching experiments were performed in the following way. First, the sample was cooled to about $40 \mathrm{~K}$ in the dark. Then, at constant temperature IF was measured irradiating the specimen for a certain time. During heating the sample to room temperature the IF spectrum was recorded. 


\subsection{Specimen}

Rectangular bars with the long axis parallel to the $\langle 110\rangle$ direction were cut from GaAs single crystals. Because of the $\overline{4} 3 m$ symmetry, stress along a $\langle 110\rangle$ axis induces a piezoelectric polarization perpendicular to this direction. The appropriate elastic and piezoelectric constants are

$$
e^{(110)}=e_{14} \text { and } c^{\langle 110\rangle}=\left(c_{11}+c_{12}+2 c_{44}\right) / 2 .
$$

$c_{11}=1.19, c_{12}=0.538$ and $c_{44}=0.595\left[10^{5} \mathrm{MPa}\right]$ are the elastic constants in the cubic system [16]. With $e_{14}=0.16 \mathrm{C} / \mathrm{m}^{2}[17]$ and $\varepsilon=12.85 \varepsilon_{0}$ [16] the relaxation strength according to Hutson and White [10] is given by $\Delta=e_{14}^{2} /\left(c^{(110)} \varepsilon\right)=$ $1.54 \times 10^{-3}$.

The resonance frequency of $106 \mathrm{kHz}$ requires a sample length of $22.4 \mathrm{~mm}$. The cross-section of the crystal was $3.0 \times 3.0 \mathrm{~mm}^{2}$. The samples are undoped SI $n$-type GaAs grown by the liquid encapsulated Czochralski method. High resistivity material is necessary to avoid the recovery of the metastable EL2* by free conduction carriers [6]. The free carrier concentration $n$ and the mobility $\mu$ at room temperature are $n=10^{7} \mathrm{~cm}^{-3}$ and $\mu=7000 \mathrm{~cm}^{2} / \mathrm{Vs}$. The concentration of EL2 is about $10^{16} \mathrm{~cm}^{-3}$ with a low compensation rate: $N_{\mathrm{A}}-N_{\mathrm{D}}=10^{14}$ to $10^{15} \mathrm{~cm}^{-3}$. Here $N_{\mathrm{A}}$ and $N_{\mathrm{D}}$ are the corresponding concentrations of shallow acceptors and donors.

\section{Experimental results}

\subsection{High-temperature results}

In this subsection we present the IF temperature dependence above $290 \mathrm{~K}$. Figure 3a shows the damping curve and Fig. $3 \mathrm{~b}-$ the corresponding frequency data. The peak in Fig. 3a has been proved to be of piezoelectrical origin. A sample coated with a thin indium layer shows a total reduction of the damping due to the short-circuit of the piezoelectric field. According to Eq. (1) we expect the peak height to be $Q_{\max }^{-1}=\Delta / 2=0.77 \times 10^{-3}$ which is in good agreement with the observed $Q_{\max }^{-1}=(1.2 \pm 0.15) \times 10^{-3}$. The experimental value is higher because of additional losses like energy dissipation in the glue, problems of contacting the quartz and of losses in the complex circuit of the apparatus.

Figure $3 \mathrm{~b}$ shows for temperatures above the peak temperature the softening of the sample due to an effective screening of the piezoelectric field. The general decrease in the frequency with rising temperature results from the temperature dependence of the elastic constants. The maximum change in the resonance frequency can be estimated from the graph to be $\delta \nu_{\max }=(50 \pm 4) \mathrm{Hz}$ for the composite oscillator. Considering the masses of the quartz and the specimen we calculate a relative frequency change $\delta \nu_{\max } / \nu=(0.87 \pm 0.07) \times 10^{-3}$ for the specimen. This agrees very well with the expected value of $\Delta / 2$.

The solid line in Fig. 3a is a Debye fit according to Eqs. (1), (2) and represents well the experimental data. From the fit we obtain the preexponential factor $\tau_{0}$ and the activation enthalpy

$$
\tau_{0}=2 \times 10^{-18 \pm 0.3} \mathrm{~s}, \quad E=(0.76 \pm 0.02) \mathrm{eV} .
$$



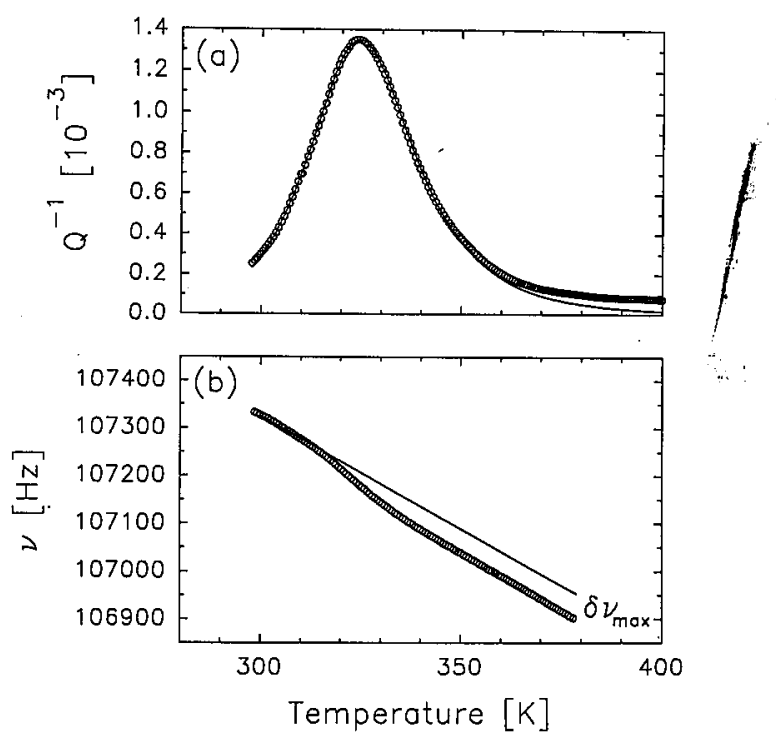

Fig. 3. IF peak (a) and fundamental frequency (b) as a function of temperature. The solid line in (a) is a Debye fit and in (b) a linear extrapolation of the low temperature data.

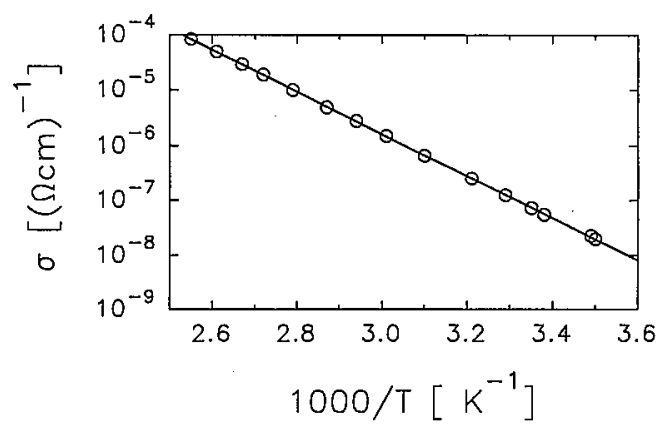

Fig. 4. Arrhenius plot of the electrical conductivity.

$E$ is the well-known energy level of EL2. To show that the observed relaxation process is of Maxwell type, Hall effect measurements were carried out to obtain the temperature dependence of the conductivity $\sigma$. The results are shown in Fig. 4 . $\sigma$ is given by

$$
\sigma=n e \mu=\sigma_{0} \exp (-U / k T) .
$$

$U$ is the energy level of the deep center. The exponential factor mainly determines the temperature dependence of the free electron concentration $n$. $e$ is the elementary charge and $\mu-$ the electron mobility. $\sigma_{0}$ is defined by Eq. (5) which by Hall measurements turned out to be temperature independent in the plotted range. 
The experimental results are

$$
\sigma_{0}=5 \times 10^{5}(\Omega \mathrm{cm})^{-1}, \quad U=0.76 \mathrm{eV} .
$$

From $\tau_{0}=\varepsilon / \sigma_{0}$ one gets $\tau_{0}=2 \times 10^{-18} \mathrm{~s}$. These values are equal to the ones obtained from the IF peak. Therefore, the relaxation time can be identified with the Maxwell time. Using this time in Eq. (1) the conductivity can be obtained from the IF data as shown in Fig. 5. For an unambiguous estimation the two cases $\omega \tau \ll 1$ and $\omega \tau \gg 1$ must be distinguished by the observation of the resonance frequency.

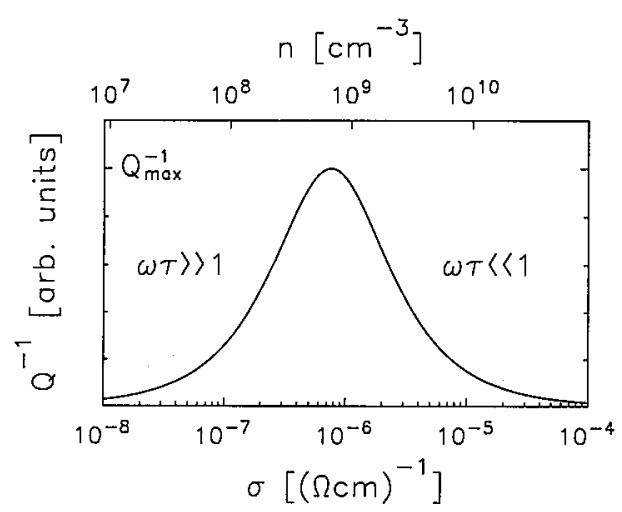

Fig. 5. IF calculated as a function of conductivity assuming a Maxwell relaxation. The carrier concentration $n$ is evaluated for a mobility of $7000 \mathrm{~cm}^{2} / \mathrm{Vs}$.

We further investigated the influence of light on the piezoelectric peak and the resonance frequency. Experimental results are shown in Figs. 6a and 6b. The solid line is achieved without exposure to light. The dotted and dashed lines show results of an irradiated sample at low $\left(I_{1}\right)$ and high $\left(I_{2}\right)$ light intensity.

To interpret these results we have to consider two quantities which contribute to the relaxation time: temperature and light intensity. At a fixed (low) temperature rising light intensity increases the conductivity by additional optically excited electrons from EL2. Thus a comparable peak is obtained by a plot of IF versus light intensity as shown by Ogawa [18] and Turek [14].

At room temperature for low light intensity the concentration of conduction electrons is slightly higher than for the unirradiated sample. For temperatures lower than the peak temperature this is shown by a small softening of the crystal. With increasing temperature the contribution of optically excited electrons becomes less compared to the total number of conduction electrons. The IF and frequency curves are approaching the data of the dark sample. For the high intensity $I_{2}$ the electron concentration at room temperature is large enough for a nearly efficient screening $(\omega \tau<1)$. Thus, with increasing temperature up to the peak temperature $Q^{-1}$ remains nearly constant. In this temperature range the portion of thermally excited electrons can be neglected. 

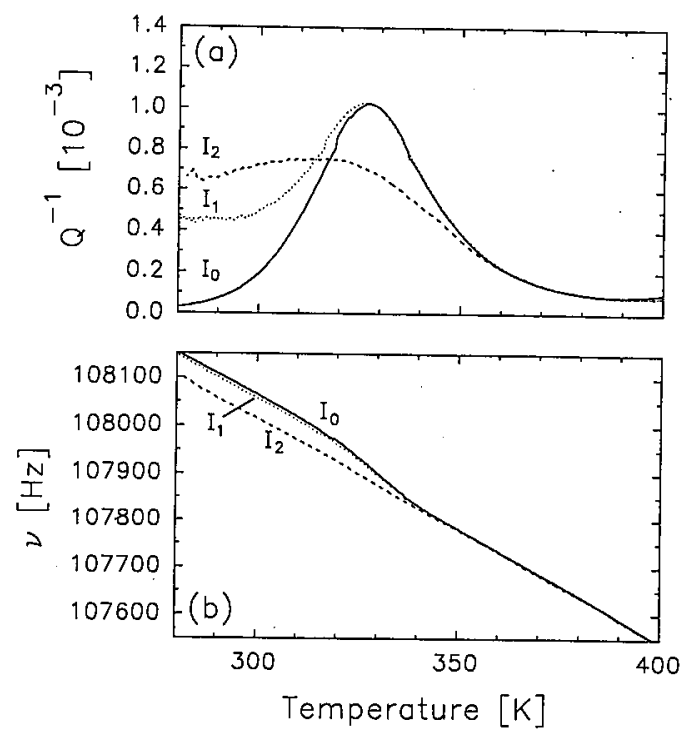

Fig. 6. IF peak (a) and corresponding frequency curves (b) for an illuminated sample and different illumination intensities $\left(I_{0}=0, I_{2}>I_{1}\right)$.

Contrary to this result Mitrokhin et al. [12] found a general suppression of the peaks by illumination with white light. We can only agree with them for high illumination intensities when $\omega \tau<1$.

\subsection{Low-temperature results}

The sample was cooled in the dark to a temperature of about $40 \mathrm{~K}$ and then heated up again to room temperature. In the whole temperature range no damping phenomena were found. The IF signal was smaller than a few $10^{-5}$.

All damping phenomena described in the following are due to acoustoelectric coupling, since all the IF signals disappear for indium rาated samples.

First, the specimen was illuminated with the Nd:YAG laser at a constant temperature of about $40 \mathrm{~K}$. Figure 7 a shows the recorded IF signal as a function of time $t$ and Fig. 7b - a corresponding plot of the frequency. Light on and off is indicated by $I(t)$, where $I=1$ means light is on (Fig. $7 \mathrm{c}$ ).

At the time $t=0$ when light is switched on we observe a very narrow damping peak (1) accompanied by a reduction of the resonance frequency. Compared with the results in Sec. 3.1 this is the screening of the electric field by a fast increase in the concentration of optically excited electrons $(\omega \tau \ll 1)$. Turning off the light at that time the same peak appears as shown in Fig. 8. The electrons are released to EL2 $(\omega \tau \gg 1)$. This process is reversible. Leaving light on for a longer period (Fig. 7) we observe a second peak (2) accompanied by an increase in the resonance frequency up to its initial value, i.e., a decrease in the conductivity. If the light is switched off in the final stage, only a weak change in $Q^{-1}$ is observed. This 

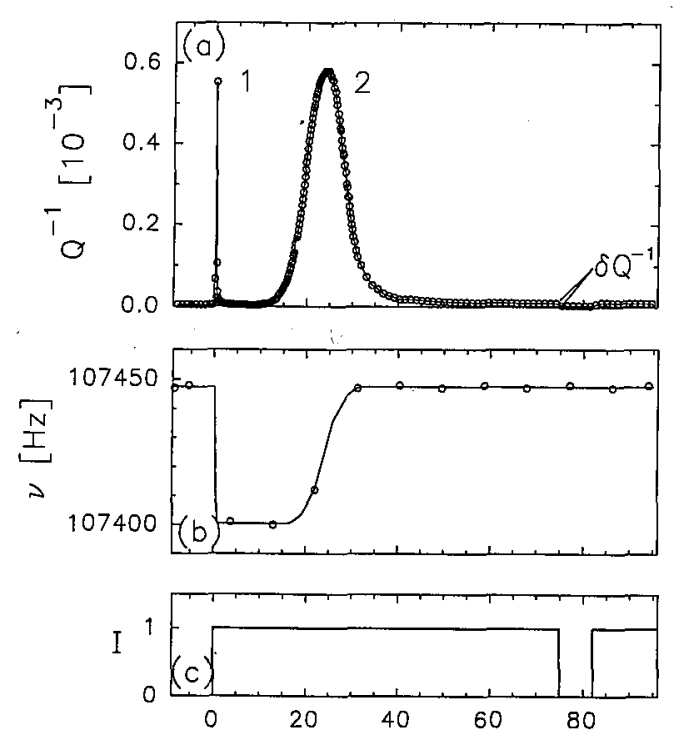

Fig. 7

Time $[s]$
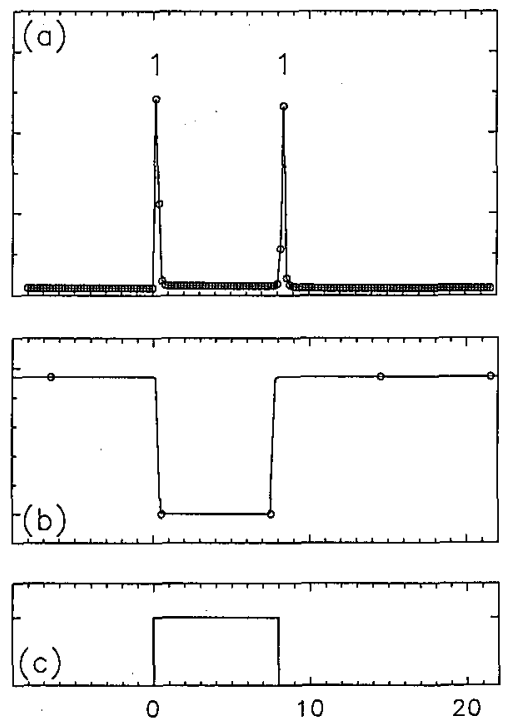

Fig. 8 Time $[\mathrm{s}]$

Fig. 7. Quenching of the light induced screening of piezofields: IF (a) and fundamental frequency (b) versus time. The solid line in (b) is obtained by several measurements. The laser was turned on at $t=0$ as indicated in (c); $T=40 \mathrm{~K}$.

Fig. 8. Reversible light induced screening of piezofields: IF (a) and fundamental frequency (b) versus time. The short time laser illumination is indicated in (c); $T=40 \mathrm{~K}$.

variation $\delta Q^{-1}$ again is reversible. Only the second peak is due to an irreversible process. The electrons must have been captured by an electrically inactive level different from EL2, because they cannot be optically excited except for a few electrons. We point out that a laser induced transformation into an electrically inactive state has occurred. More light intensity leads to a faster reaction and to a bigger difference $\delta Q^{-1}$. This indicates a somewhat higher amount of free electrons involved in the process. It was proved that a long time illumination exceeding 15 minutes does not cause any change in the signals.

Subsequent to these irradiation experiments the sample was heated up to room temperature in the dark. Figure 9 shows the damping and frequency curves for a heating rate of $6 \mathrm{~K} / \mathrm{min}$. In the temperature range above $150 \mathrm{~K}$ no more peaks were found. The solid lines show the data by cooling the sample in the dark before it was illuminated. After illumination two damping peaks were found

$$
\text { peak (I): at } 60 \pm 3 \mathrm{~K}, \text { peak (II): at } 95 \pm 3 \mathrm{~K} \text {. }
$$

Both peaks are due to acoustoelectric coupling but not comparable with the piezoelectric one above room temperature. This is shown by the behavior of the resonance frequency in Fig. $9 \mathrm{~b}$. With rising temperature the conductivity increases and carriers start to screen the piezoelectric fields. But the condition $\omega \tau=1$ at the maximum temperature $T_{\max }^{(\mathrm{I})}$ is not completely fulfilled $(\omega \tau>1$ and $\omega \tau \approx 1)$. 

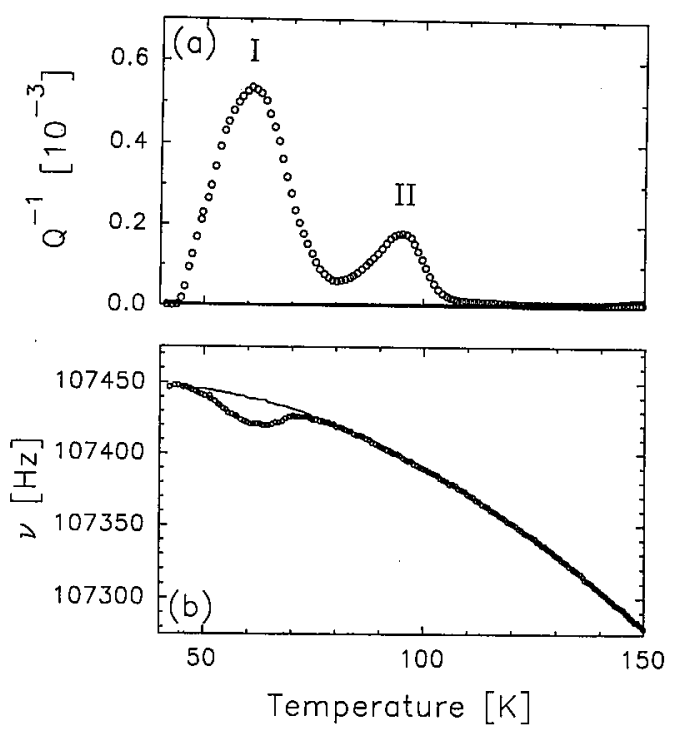

Fig. 9. IF peaks (a) and corresponding frequency data (b) for a cooled sample (solid line) and for a heated sample after photoquenching at $40 \mathrm{~K}$.

Above $T_{\max }^{(\mathrm{I})}$ the screening becomes less efficient. This is shown by the stiffness of the sample. Obviously free carriers are captured. Approaching $T_{\max }^{(\mathrm{II})}$ the conductivity rises again. The corresponding change in the resonance frequency cannot be dissolved, because the screening effect is too small. The IF peak (II) corresponds to the conductivity curve in this temperature range. For temperatures above $110 \mathrm{~K}$ the conductivity is below the detection range of the IF method $\left(n<10^{7} \mathrm{~cm}^{-3}\right)$.

A lowering of the heating rate leads to a reduction of the peak heights and to a shift of the peaks to lower temperatures. This is shown in Fig. 10.

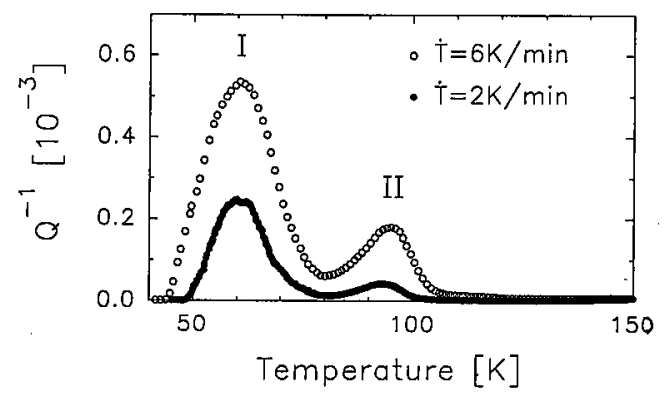

Fig. 10. IF spectra for two different heating rates $\dot{T}$. The sample was photoquenched at $40 \mathrm{~K}$.

The ratio of the peak heights is similar for different samples cut from the same crystal. A variation is observed for samples from different crystals. The maximum 
peak temperatures always are about the same.

We conclude from our illumination experiments that we observe in Fig. 9 the recovery of an electron state induced by light at $40 \mathrm{~K}$. The peaks only appear when the samples are irradiated at low temperature. The recovery becomes apparent by a release of carriers either to the valence or the conduction band.

\section{Discussion}

The acoustoelectric relaxation peaks as shown in Sec. 3 can be excellently described by a Maxwell time of dielectric relaxation. Light affects the relaxation time in the way it increases the conductivity. This is in agreement with results published by Ogawa [18], Hirano [11], and Hutson [10] but in contrast to the ones obtained by Mitrokhin et al. [12]. The latter dispute a Maxwell time, because their parameters achieved from IF measurements do not fit the conductivity. In our opinion their problem arises from changes in the bulk charge due to flexural vibrations. This bulk charge of alternating sign leads to a small variation of the electron concentration and consequently of the relaxation time. A variation of the relaxation time broadens the IF peak [19] and then the evaluated activation enthalpy will be too small as found by Mitrokhin. Since the determination of $\tau_{0}$ depends on the activation enthalpy, the calculated value of $\tau_{0}$ also will differ.

The results presented in Sec. 3.2 indicate that the conditions for the formation and the recovery of a metastable state correlate with the features of EL2*. We now want to present a model of the EL2* formation that is based on our low-temperature results. Under photon flux different optical excitation processes are possible:

- neutral (uncompensated) EL2 centers are emptied and partially refilled by electron capture according to a relation

$$
(\mathrm{EL} 2)^{0} \rightleftarrows(\mathrm{EL} 2)^{+}+\mathrm{e}^{-}
$$

holes $\mathrm{p}^{+}$:

$$
(\mathrm{EL} 2)^{+} \rightleftarrows(\mathrm{EL} 2)^{0}+\mathrm{p}^{+}
$$
$(\mathrm{EL} 2)^{+}$.

- electrons are released from negatively charged shallow acceptors $\mathrm{A}^{-}$to

The concentration of shallow donors can be neglected here. This point will be discussed later.

Due to (6) and (7), free electrons and holes are generated. These carriers are detected in our experiment by their screening of the piezoelectric field. The conductivity decreases under continuous illumination according to the following relation:

$$
(\mathrm{EL} 2)^{0} \rightleftarrows\left(\mathrm{EL}^{*}\right)^{0}
$$

After a sufficiently long time nearly all EL2 centers are transformed to EL2* and most of the free electrons are trapped. The residual EL2 defects arise from the small 
amount of partial photoinduced recovery as suggested in Eq. (8). With increasing light intensity the equilibrium is shifted to the left side, since we observe a higher conductivity (a larger $\delta Q^{-1}$ in Fig. 7).

The transition from all EL2 defects to the metastable state is sufficient to end the optical excitation of electrons into the conduction band, because the shallow donors are completely compensated by the shallow acceptors and their concentration is small compared to the other states. Therefore they do not need to be considered here. When EL2 is in its metastable state, the residual shallow acceptors can no longer be compensated by electrons from EL2. As their ionization energy is about $0.03 \mathrm{eV}$ (carbon $0.026 \mathrm{eV}$ and zinc $0.031 \mathrm{eV}$ ), free holes should be generated and these lead to a detectable conductivity. So far our picture coincides with the model proposed by Bray et al. [20], which is based on electronic Raman scattering. The generation of free holes, however, is not observed in our experiments. The conductivity is smaller than $10^{-8}(\Omega \mathrm{cm})^{-1}$ for both light on and off. Thus neither photoconductivity nor persistent photoconductivity when the light is turned off are observed. The latter was reported by Jiménez et al. [21] for SI Czochralski samples at $77 \mathrm{~K}$ after an illumination of about 10 minutes but they state that it is not a universal phenomenon. Our experimental results show that no transitions from the valence band to the acceptors are allowed. A compensation of these defects by EL2* would require donor-like states for EL2* in the gap. However, these are not generally accepted. We believe that the shallow acceptors have to undergo a transition to an electrically inactive neutral metastable state as well as EL2.

This can be understood according to a model by Jiménez et al., after it has been modified. The basic idea is to connect our IF results with their photocurrent data. Band-edge photoconductivity measurements $(h \nu \geq 1.4 \mathrm{eV})$ show that after illumination the optical transitions operating from the acceptor level are locked. This arises from an association of shallow acceptors A with EL2 defects. They both are in close neighborhood in the crystal [22]. We believe that the complex transforms to a metastable (EL2*-A $\mathrm{A}^{0}$ ) center. In opposition to Jimenez this center has to be electrically inactive as we observe no persistent photoconductivity. It is well known that variations in the properties of midgap levels are related to different crystal growth conditions or thermal history of the samples. Therefore this contradiction should not be surprising.

According to the above considerations the photoquenched material shows two different metastable states: the (EL2*-A $\left.{ }^{0}\right)$ complex and the "single" EL2*. The recovery of these states can be discussed in terms of our heating experiments. If the samples are heated up to temperatures above $120 \mathrm{~K}$, we observe two damping peaks due to local maxima in the conductivity. These indicate the recovery of two metastable defects. The dependence of the IF peaks on the heating rate $\dot{T}$ (Fig. 10) unambiguously reveals a transformation reaction (for $\dot{T}=0$ the IF decays to zero). In Fig. 9 we assign peak (I) to the recovery of the (EL2*-A $\left.{ }^{0}\right)$ center, because the recovery of EL2* is observed at higher temperatures [6]. At about $60 \mathrm{~K}$ the complex dissociates. The recovered acceptors are thermally ionized by electrons from the valence band. This generation of free holes increases the conductivity. The regeneration process must be coupled to a partial recovery of EL2* because of the 
following decrease in the conductivity. The normal states of EL2 then compensate the shallow acceptors.

The thermal recovery of EL2* is indicated by peak (II) at $95 \mathrm{~K}$. It is coupled with an electronic transition releasing free carriers. Electrons are emitted and then captured from regenerated normal states of EL2. Thus we assume the transformation EL2 ${ }^{*} \longrightarrow$ EL2 to occur via the charged state (EL2) ${ }^{+}$.

The recovery temperature is approximately $20 \mathrm{~K}$ smaller than the one observed from other experiments. Our method registers the temperature of maximum electron concentration, which not necessarily coincides with the temperature of maximum regeneration rate.

The varying ratio of the peak heights (I/II) for different crystals now can be understood by different compensation ratios: a larger concentration of shallow acceptors will result in a higher peak (I). On account of the coupling to EL2* more of these defects will recover. Consequently, fewer EL2* recover at the higher temperature and peak (II) becomes smaller.

In summary, we have shown that EL2 and shallow acceptors are transferred to neutral metastable states under a photon flux of $1.17 \mathrm{eV}$. The acceptor-EL2* complex and EL2* recover for temperatures above 60 and $95 \mathrm{~K}$ respectively. Both formation and recovery are accompanied by electronic transitions. Very small conductivities in the range of $10^{-7}(\Omega \mathrm{cm})^{-1}$ are detected by the IF technique indicating a recovery of EL2* through (EL2) ${ }^{+}$.

We thank Deutsche Forschungsgemeinschaft for financial support.

\section{References}

[1] H.J. von Bardeleben, D. Stiévenard, D. Deresmes, A. Huber, J.C. Bourgoin, Phys. Rev. $B$ 34, 7192 (1986).

[2] T. Figielski, T. Wosiński, Phys. Rev. B 36, 1269 (1987).

[3] D.J. Chadi, K.J. Chang, Phys. Rev. Lett. 60, 2187 (1988).

[4] J.F. Wager, J.A. Van Vechten, Phys. Rev. B 35, 2330 (1987).

[5] B.K. Meyer, D.M. Hofmann, J.R. Niklas, J.-M. Spaeth, Phys. Rev. B 36, 1332 (1987).

[6] G. Vincent, D. Bois, A. Chantre, J. Appl. Phys. 53, 3643 (1982).

[7] J.C. Bourgoin, H.J. von Bardeleben, D. Stiévenard, J. Appl. Phys. 64, R65 (1988).

[8] S. Nojima, J. Appl. Phys. 58, 3485 (1985).

[9] G.M. Martin, Appl. Phys. Lett. 39, 747 (1981).

[10] A.R. Hutson, D.L. White, J. Appl. Phys. 33, 40 (1962).

[11] R. Hirano, Y. Kawai, T. Ogawa, Jpn. J. Appl. Phys. 24, 1049 (1985).

[12] V.I. Mitrokhin, S.I. Rembeza, V.V. Sviridov, N.P. Yaroslavtsev, Phys. Status Solidi A 119, 535 (1990).

[13] C. Zener, Elasticity and Anelasticity of Metals, Univ. of Chicago Press, Chicago 1948.

[14] I. Turek, Phys. Status Solidi A 9, 113 (1972).

[15] R.B. Schwarz, Rev. Sci. Instrum. 48, 111 (1977). 
[16] J.S. Blakemore, J. Appl. Phys. 53, R123 (1982).

[17] G. Arlt, P. Quadflieg, Phys. Status Solidi 25, 323 (1968).

[18] T. Ogawa, J. Phys. Soc. Jpn. 17, 400 (1962).

[19] R. de Batist, Internal Friction of Structural Defects in Crystalline Solids, North-Holland, Amsterdam, London 1972, p. 75.

[20] R. Bray, K. Wan, J.C. Parker, Phys. Rev. Lett. 57, 2434 (1986).

[21] J. Jiménez, P. Hernández, J.A. de Saja, J. Bonnafé, Phys. Rev. B 35, 3832 (1987).

[22] E.J. Johnson, J. Kafalas, R.W. Davies, W.A. Dyes, Appl. Phys. Lett. 40, 993 (1982). 